\title{
À propos de quelques structures polyprédicatives comparatives à dominante extensive et/ou intensive
}

\author{
About several polypredictive comparative structures \\ with extensive and/or intensive dominant
}

\author{
Alicja Hajok \\ Université Pédagogique de Cracovie, Pologne \\ alicja.hajok-kornas@up.krakow.pl
}

\begin{abstract}
The objective of this study is to discuss the semantic properties of the comparative structures polyprédicatves to dominant extensive and/or intensive. The comparative structures released for the need for this study make it possible to specify the nature of the concept of 'compariso'.
\end{abstract}

Keywords: comparison, extension, intensification, quantification, lexico-syntactic structure, predicative structure

L'objectif de ce travail est de discuter quelques propriétés sémantiques (i) des structures polyprédicatives comparatives à dominante extensive et/ou intensive et (ii) des structures dites fausses comparatives. Les structures dégagées pour le besoin de cette étude nous permettent de préciser la nature du concept de « comparaison » ainsi que de classer la comparaison comme un hyperprédicat.

\section{INTRODUCTION}

À titre d'introduction, nous dressons trois constats :

(i) Les travaux sur la comparaison ne manquent pas. Les études consacrées à cette question sont souvent mises en relation avec les sujets apparentés 
comme gradation, scalarité, intensification. Dans la littérature de sujet, nous observons deux approches classifiant les relations comparatives : (a) traditionnellement, la question de la comparaison est évoquée lors des descriptions liées à la gradation des adjectifs qualificatifs ou des adverbes à valeur qualificative ainsi la comparaison est vue par le prisme de trois relations : supériorité, égalité, infériorité, (b) les études récentes (p.ex. R. Rivara, $\mathrm{C}$. Fusch, $\mathrm{Cl}$. Muller) soutiennent une thèse selon laquelle dans les langues indo-européennes n'existent que deux relations comparatives : égalité et inégalité. La présente étude retient cette thèse en précisant ainsi que la comparaison d'inégalité exprime (i) la variation d'une qualité prédicative vers le plus ou le moins ou (ii) la variation d'une quantité vers le plus ou le moins. Quant à la comparaison d'égalité, on observe la simultanéité sur la variation d'une qualité ou d'une quantité A et B. Alors, si nous avons deux objets A et $\mathrm{B}$ qui représentent soit une grandeur extensive soit une grandeur intensive, nous pouvons les comparer selon un trait commun : $p$ pour des grandeurs extensives et $q$ pour des grandeurs intensives. La comparaison résulte en jugement d'égalité ou en jugement d'inégalité entre $\mathrm{A}$ et $\mathrm{B}$ selon le critère $p$ ou $q$ : comparer $A$ et $B$ selon le trait $p$ (ou $q$ ) induit pour $A$ et $B$ qu'ils sont égaux / inégaux selon le critère $p$ (ou q).

(ii) Une définition simple et univoque du concept de 'comparaison' demande de délimiter le champ d'investigation de cette étude. Nous limitons nos analyses aux constructions comparatives à complément nominal : Luc a bu autant de vin que Stan.

(iii) Le nom occupe une place particulière dans les travaux de linguistique. Il existe de nombreuses études consacrées à l'analyse de propriétés spécifiquement nominales, portant sur la distinction entre noms propres et noms communs, l'opposition entre noms massifs et noms comptables, ou entre noms de matières et noms d'abstraction. Les grammaires traditionnelles retiennent souvent la caractérisation ontologique des noms en les divisant en noms concrets et en noms abstraits. G. Kleiber et M. Vuillaume (2011) précisent que cette division peut « correspondre à celles entre référents (i) matériels et immatériels, (ii) accessibles et inaccessibles aux sens, (iii) autonomes et dépendants ontologiquement, i.e. dont l'existence présuppose ou non celle d'une autre entité. Les trois définitions ne se recouvrent pas ». Pour le besoin de cette étude, nous faisons recours aux thèses défendues par D. Van de Velde (1995) et N. Flaux, D. Van de Velde (2000) qui proposent de classifier les noms en noms de matières et en noms d'abstraction. Étant donné qu'on a démontré (D. Van de Velde, 1995) la différence entre les deux types de grandeurs, celles qu'on attribue aux choses et celles qu'on attribue aux qualités des choses, il parait justifiable de discuter les critères selon lesquelles se fait la comparaison quantitative et la comparaison qualitative. 


\section{PRÉALABLES MÉTHODOLOGIQUES}

Nous appuyons ce travail sur les principes méthodologiques retenues par la grammaire à base sémantique qui sont les suivantes : la structure prédicative constitue l'unité minimale d'analyse ; la proposition est constituée d'un prédicat et de ses arguments; on distingue les prédicats monovalents qui impliquent un seul argument et les prédicats polyvalents qui impliquent au moins deux arguments; vu la nature des arguments impliqués : les arguments élémentaires et les arguments prépositionnels, on distingue les prédicats de premier ordre et les prédicats d'ordre supérieur.

\section{CONCEPT DE «COMPARAISON »}

Au niveau sémantique, nous voyons le concept de «comparaison » comme une structure polyprédicative, composée des éléments suivants : un prédicat de comparaison hiérarchiquement supérieur au prédicat obligatoirement graduable, un prédicat graduable employé par rapport à deux arguments non coréférents, par exemple Luc est moins fier que Stan réalise le schéma prédicatif suivant: \{moins_que [fier (Luc)] [fier (Stan)]\} où :

(i) le prédicat de comparaison (moins_que) est hiérarchiquement supérieur au prédicat obligatoirement graduable (fier), mais pas nécessairement noté à la surface de la phrase. Comparons: Luc est aussi fier que Stan vs Luc est comme Stan et Luc est moins fier que Stan vs Luc n'est pas comme Stan.

(ii) le prédicat de comparaison ouvre deux positions argumentales obligatoirement saturées par des arguments différents mais de même nature ( $L u c$, Stan), c'est-à-dire le comparé peut devenir le comparant et l'inversement. Comparons : Luc est moins fier que Stan vs Stan est plus fier que Luc, contrairement à Luc est fier comme un pou et *Un pou est fier comme Luc. La pertinence de ce procès était déjà remarquée par J.-J. Rousseau (Rousseau, in Lafaye, Dict. des synonymes, p. 948) qui dans sa définition avait dressé une structure profonde de «comparaison »: «[...] Par la comparaison, je remue les objets, je les transporte pour ainsi dire, je les pose l'un sur l'autre pour prononcer sur leur différence ou sur leur similitude », en bref on retient : le prédicat d'action - de mouvement (je remue les objets, je les transporte), le prédicat d'action - de dire (prononcer) et le prédicat d'état - de quantité ou de qualité (leur différence ou leur similitude), sur lesquels s'impose le prédicat d'ordre supérieur de nature résultative qui dénote un état et qui résulte de l'action dénoté par le verbe comparer. Par le fait de comparer, deux objets $\mathrm{A}$ et $\mathrm{B}$ sont mis en relation, l'objectif est de trouver la correspondance entre ces deux objets de pensée vus à la fois comme identiques et différents. Comparons : 
1) Luc compare les deux chaises.

(a) Luc compare la fonctionnalité de deux chaises.

(i) Luc compare la fonctionnalité de la chaise_1 avec la chaise_2.

2) Luc compare les deux chaises pour se prononcer sur leur fonctionnalité.

(a) Luc compare les deux chaises pour dire si la chaise_l est plus / moins / aussi fonctionnelle que la chaise_2.

Ainsi le modèle sémantique du concept de «comparaison » se présente comme une structure polyprédicative :

Action_1 $\left\{\mathrm{N}<\right.$ hum $\left.>, \mathrm{f}\left(\mathrm{x}_{1}\right), \mathrm{f}\left(\mathrm{x}_{2}\right)\right\}$ CAUSE Action_2 $\left\{\mathrm{N}<\right.$ hum $\left.>, \mathrm{f}\left(\mathrm{x}_{1}\right) \cap \mathrm{f}\left(\mathrm{x}_{2}\right)\right\}$ RÉSULTAT État \{comparaison $\left[\mathrm{f}\left(\mathrm{x}_{1}\right)=\mathrm{f}\left(\mathrm{x}_{2}\right) \mathrm{U} \mathrm{f}\left(\mathrm{x}_{1}\right)>\mathrm{f}\left(\mathrm{x}_{2}\right) \mathrm{U} \mathrm{f}\left(\mathrm{x}_{1}\right)<\mathrm{f}\left(\mathrm{x}_{2}\right)\right]$ \}

Cette structure peut, mais ne doit pas être saturée à la surface de la phrase.

\section{STRUCTURES POLYPRÉDICATIVES COMPARATIVES À DOMINANTE EXTENSIVE}

Il existe des groupes sémantiques (Wierzbicka, 1971), dans lesquels le comparativus a un caractère primaire, par exemple : les mesures de l'espace où les rapports sont mesurables et quantifiables, par exemple : petit / grand, long / court, large / étroit, etc. De cette constatation, nous retenons le lexème quantifiable qui nous renvoie par sa dénomination à la quantification, ainsi qu'à la notion de quantité. Pour ne pas chercher trop loin, nous proposons d'analyser des phrases forgées sur le déterminant nominal une quantité de : (i) Luc a une quantité de livres, (ii) Luc a une grande / petite quantité de livres. Une quantité de livres dans la plupart des cas équivaut à une grande quantité de livres, mais l'ajout de l'adjectif petit est nécessaire pour exprimer la petite quantité (Flaux, Van de Velde, 2000). Quant à l'adjectif grand, il semble être facultatif. Cependant, en inscrivant cette phrase dans une relation de comparaison, il est nécessaire de mettre en jeu les adjectifs qualificatifs qui modifient le déterminant nominal : *Luc a une quantité de livres que Stan vs Luc a une plus petite quantité de livres que Stan et Luc a la même quantité de livres que Stan. La comparaison ne porte ni sur Luc et Stan, ni sur les livres. La relation de comparaison porte sur la quantité de livres.

Les noms d'actions sont vus comme les noms abstraits extensifs (Flaux, Van de Velde, 2000, p. 97), car ils ont une étendue temporelle. Nous parlons ainsi de l'extension qui est comprise comme «l'étendue spatiale ou temporelle. Sa description explique la compatibilité des $\mathrm{N}$ avec les prédicats dimensionnels, comme dans Le $N$ mesure $x$ espace, Le $N$ a duré $x$ temps, un $N$ de $\{x$ espace / $x$ temps $\}$, $\{x$ espace $/ x$ temps $\}$ de $N$, où $x$ espace et $x$ temps sont des expressions constituées d'un déterminant numéral et d'un nom d'unité de mesure spatiale ou temporelle (e.g. trois hectares, cinq centimètres, deux heures, dix ans) » (Huyghe, 2015, p. 9). 
Empruntons donc un exemple à D. Van de Velde (2000, p. 100) : marcher est un verbe d'activité et marche est un nom d'activité qui bénéficie de l'étendue temporelle de nature fréquentative (i), durative (ii), qualitative (iii) et quantifiable (iv). La relation de comparaison porte respectivement sur la fréquence, la durée, la qualité et la quantité.

(i) $\mathrm{Q}$ comparaison inférée HYPERPRÉDICAT $\left\{\mathrm{P}_{\text {FREQ (souvent)Prédicat du second ordre }}[\mathrm{R}\right.$ marche $\left.(\mathrm{x})]\right\}$

Luc marche beaucoup

Luc marche plus que Stan

Luc marche plus souvent que Stan

Luc a fait beaucoup plus de marche que Stan

Luc a fait beaucoup moins de marche que Stan

(ii) $\mathrm{Q}$ comparaison inférée HYPERPRÉDICAT $\left\{\mathrm{P}\right.$ DURATIF (longtemps)Prédicat du second ordre $\left.\left[\mathrm{R}_{\text {marche }}(\mathrm{x})\right]\right\}$

Luc marche très longtemps

Luc marche plus longtemps que Stan

Luc a fait une marche plus longue que Stan

(iii) $\mathrm{Q}$ comparaison inférée HYPERPRÉDICAT $\{\mathrm{P}$ qualitative (mieux)Prédicat du second ordre $[\mathrm{R}$ marche $(\mathrm{x})]\}$

Luc marche très bien

Luc marche mieux que Stan

La marche de Luc est meilleure que celle de Stan

(iv) $\mathrm{Q}$ comparaison inférée HYPERPRÉDICAT $\left\{\mathrm{P}_{\text {quantifiable (rapidement) Prédicat du second ordre }}\left[\mathrm{R}_{\text {marche }}(\mathrm{x})\right]\right\}$

Luc marche très rapidement

Luc marche plus rapidement que Stan

La marche de Luc est plus rapide que celle de Stan

\section{STRUCTURES POLYPRÉDICATIVES COMPARATIVES À DOMINANTE INTENSIVE}

Nous sortons du point de vue que l'intensification est « une intervention du locuteur qui consiste à amplifier ou à amoindrir le degré d'une certaine propriété » (Adler et Asnès, 2013, p. 10). Nous avons déjà discuté la notion d'intensité par rapport aux degrés de significations des adjectifs qualificatifs, par exemple : Luc est très rusé. Nous avons posé une hypothèse que « si l'intensité se présente comme un prédicat de second ordre qui prend pour argument un énoncé, alors que la comparaison se superpose comme un prédicat hiérarchiquement supérieur qui prend pour argument deux énoncés. Ainsi, on doit faire face à la description de la structure polyprédicative dont la première valeur sémantico-syntaxique renvoie à la comparaison et la seconde valeur sémantique renvoie à l'intensification » (Hajok, 2017). La 
représentation syntaxico-sémantique de cette structure prédicative est la suivante :

$$
\mathrm{Q} \text { comparaison inférée HYPERPRÉDICAT }\left\{\mathrm{P}_{\text {inf (très)Prédicat du second ordre }}\left[\mathrm{R}_{\text {rusé }}(\mathrm{x})\right]\right\}
$$

Car la proposition Luc est très rusé doit être interprétée ainsi : Je compare Luc aux autres gens. Je compare la ruse de Luc à la ruse des autres personnes.

D. Van de Velde (1995, p. 131) précise que toutes les grandeurs intensives sont des qualités ou des états. Les noms d'abstraction sont successibles d'augmenter (INT+) et de diminuer (INT-) et pour cela ils s'imposent aux relations comparatives à dominante intensive, par exemple : Tu as beaucoup de chance de réussir. Tu as plus de chance de réussir que ton frère. Très et beaucoup de sont des exposants de l'intensité appropriés respectivement aux adjectifs et aux noms abstraits. Ces deux propositions ont la même représentation syntaxico-sémantique et elles bénéficient de la même interprétation : la première valeur sémantico-syntaxique renvoie à la comparaison et la seconde renvoie à l'intensification.

Mais une telle description est mise en doute lorsqu'on parle des noms d'abstractions renvoyant aux attitudes intellectuelles, psychologiques et morales, et encore d'autres. T. Muryn $(2005$, p. 79$)$ remarque que patience est graduable en termes d'intensité. Elle souligne que la patience se relève par fragments. "Pour quelqu'un qui est très patient ou bien qui a beaucoup de patience, la progression qui oscille entre les intervalles de patience et d'impatience démontre le nombre beaucoup plus élevé de ces premiers que de seconds» (Muryn, 2005, p. 79). L'intensité devrait être décrite en termes de haute ou basse fréquence. Ainsi l'exemple proposé par T. Muryn (2005, p. 80) Il a beaucoup plus de patience que toi devra être interprété comme Il est plus souvent patient que toi donc il ne s'agit pas de structure à dominante intensive mais à dominante extensive.

\section{LES MARQUEURS DE COMPARAISON ET LE MÉCANISME INTENSIFICATOIRE}

Certaines structures comparatives sont abordées par les écrits linguistiques et par les grammaires tantôt dans le chapitre sur la comparaison tantôt dans le chapitre sur l'intensification, ce qui provoque une certaine ambiguité dans leur classification. P.-A. Buvet et G. Gross $(1995$, p. 83) précisent à propos des structures $N A D J$ comme $N$ (les yeux bleus comme la turquoise, les yeux bleus comme des myosotis) qu'elles « n'ont pas une interprétation comparative mais expriment l'intensité ou le haut degré » et de même pour les structures NdeN : la patience d'ange, la faim de loup, etc. où le $d e N$ fonctionne comme modifieur intensif.

Ces constructions sont appelées dans la littérature de sujet les comparaisons à parangon ou les comparaisons stéréotypées. Nous faisons recours à la langue polonaise pour montrer que les comparaisons à parangon sont souvent de nature el- 
liptique. Prenons comme l'exemple la construction $N$ jak $N$ ( $N$ comme $N)$ : oczy jak chabry (yeux-comme-bleuets), oczy jak niezapominajki (yeux-comme-myosotis). Le marqueur de comparaison jak (comme) met en relation les deux $\mathrm{N}$, mais la comparaison ne porte pas sur les N, mais sur la qualité de N. Dans cette structure, le comparé est inféré. Dans Maria ma oczy jak chabry (Marie a les yeux comme bleuets) il ne s'agit pas de comparer oczy (yeux) à chabry (bleuets), mais le couleur bleu des yeux avec le couleur bleu des fleurs. Cette qualité n'est pas exprimée à la surface de la phrase. De plus la valeur stéréotypée attribuée aux bleuets intensifie le couleur bleu.

Maria ma oczy jak chabry [Marie-a-yeux-comme-bleuets]

Jej oczy sa jak chabry [Ses-yeux-sont-comme-bleuets]

Oczy sq niebieskie i chabry sq niebieskie [Ses-yeux-sont- bleus-et-bleuets-sont-bleus]

Chabry maja wyjatkowo niebieski kolor [Bleuets-ont-exceptionnel-bleu-couleur]

Oczy sa bardzo niebieskie [Ses-yeux-sont- très-bleus]

Il s'agit donc de «fausses comparatives où l'expression du haut degré repose sur l'association de la propriété considérée avec un terme pris comme le parangon ou le stéréotype de cette propriété » (Buvet et Gross, 1995, p. 84). Ces constructions sont forgées sur l'idée de comparaison, mais qui ne réalisent pas le concept de « comparaison» défini plus haut (cf. 3 Concept de "comparaison»). Le test de l'effacement et le test de la substitution du modifieur qui renvoie au prédicat nominal le prouvent $(1,3)$. Cependant, quand le modifieur renvoie à l'argument $(2,4)$ son effacement fait apparaître le prédicat graduable qui renvoie à la propriété stéréotypée. Dans les deux cas, les modifieurs sont semi-figés ou figés et appropriés au N.

(1) Luc a une faim de loup

Effacement : Luc a faim

Substitution : Luc a très faim

(2) Marie a une taille de guêpe

Effacement : ?? Marie a une taille

Substitution : Marie a une taille très fine

(3) Lukasz ma anielska cierpliwość [Luc-a-d'ange-patience]

Effacement : Łukasz ma cierpliwość [Luc-a-patience]

Substitution : Łukasz ma wielka / ogromna cierpliwość [Luc-a-grand / énorme-patience]

(4) Maria ma oczy jak chabry [Marie-a-yeux-comme-bleuets]

Effacement : ?? Maria ma oczy [Marie-a-yeux]

Substitution : Maria ma bardzo blękitne oczy [Marie-a-très-bleus-yeux]

Il est nécessaire donc de distinguer l'opérateur de comparaison (i) de l'opérateur d'intensification (ii), cependant tous les deux sont fondés sur le même marqueur de comparaison, par exemple comme et jak: (i) Luc est bête comme ses pieds (Lukasz 
jest glupi jak but) et *Les pieds de Luc sont bêtes (Buty Lukasza sa głupie) vs (ii) Luc est comme Stan (Lukasz jest jak Staś) et Stan est comme Luc (Staś jest jak tukasz).

Certaines constructions peuvent avoir une double interprétation, celle de comparaison ou celle d'intensification qui dépend du contexte dans lequel elles apparaissent, par exemple : riche comme Crésus ou fort comme un Turc.

Luc est riche comme Crésus (Lukasz jest bogaty jak Krezus) $\rightarrow$ marqueur de comparaison: comme (jak) $\rightarrow$ opérateur de comparaison: COMPARAISON D’ÉGALITÉ (quantité) $\rightarrow$ interprétation : Luc a autant d'argent que Crésus (Lukasz ma tyle samo pieniędzy, co Krezus).

Luc est riche comme Crésus (Lukasz jest bogaty jak Krezus) $\rightarrow$ marqueur de comparaison: comme (jak) $\rightarrow$ opérateur d'intensification: INT+ (qualité) $\rightarrow$ interprétation : Luc est très riche (Lukasz jest bardzo bogaty).

\section{CONCLUSION}

Cet article aborde quelques aspects sémantiques du concept de « comparaison », en se concentrant sur des structures polyprédicatives comparatives de nature intensive et/ou extensive. En dépit des problèmes qu'elle met en lumière, la distinction entre $\mathrm{N}$ de matières et $\mathrm{N}$ abstraits se détecte au niveau de la réalisation de la structure polyprédicative comparative, et cela par l'emploi des lexèmes en position prédicative accompagnés d'une construction évoquant l'intensité et/ou l'extensité. Le prédicat de « comparaison » prévaut sur cette construction. 


\section{BIBLIOGRAPHIE}

Adler, S., Asnes, M. (2013). «Qui sème la quantification récolte l'intensification ». In : Langue française, $\mathrm{n}^{\circ} 177,9-22$.

Buvet, P.-A., Gross, G. (1995). « Comparaison et expression du haut degré dans le groupe nominal ». In : Faits de langues, ${ }^{\circ}$ 5. La comparaison, 83-88. DOI : https://doi.org/10.3406/ flang.1995.979; www.persee.fr/doc/flang_1244-5460_1995_num_3_5_979.

Dictionnaire des synonymes de la langue française (1858). Lafaye, Edité par Hachette.

Flaux, N., Van De Velde, D. (2000). Les noms en français. Esquisse de classement. Édition Ophrys.

Fuchs, C. (2014). La comparaison et son expression en français. Editions Ophrys.

Guimier, C. (2007). «L'adverbe tout en construction comparative : tout prémodifieur de aussi». In : Verbum, XXIX, 3-4, 273-286.

Hajok, A. (2017). « À propos de structures polyprédicatives du type comparatif ». In : Synergies Pologne, $\mathrm{n}^{\circ} 14$.

Hajok, A. (2018). «Le prédicat comparer et le concept de comparaison ». In : Сборник статей по итогам III-й международной конференции « Язык и действительность. Научные чтения на кафедре романских языков им. В.Г. Гака». Moskwa, p-ISBN : 978-5-99734923-3, 427-431.

Huyghe, R. (2015). «Les typologies nominales : présentation ». In : Langue française, $\mathrm{n}^{\circ} 185$, 5-27. DOI : 10.3917/lf.185.0005. URL : https://www.cairn.info/revue-langue-francaise-20151-page-5.htm.

Kleiber, G., Vuillaume, M. (2011). « Sémantique des odeurs ». In : Langages, 181, 17-36.

Legallois, D., Tutin, A. (2013). « Vers une extension du domaine de la phraséologie ». In : Langages, 189. Armand Colin.

Leroy, S. (2003). « Les constructions comparatives intensives dans Illusions perdues ». In : L'Information Grammaticale, $\mathrm{n}^{\circ}$ 99, 39-42. DOI : https://doi.org/10.3406/igram.2003.2602;www. persee.fr/doc/igram_02229838_2003_num_99_1_2602.

Muller, C. (1996). La subordination en français. Paris : Armand Colin.

Muryn, T. (2005). « Continuité, intensité et fréquence ». In : Synergies Pologne, n 2, 78-83.

Rivara, R. (1990). Le système de la comparaison. Sur la construction du sens dans les langues naturelles. In : Les Éditions de Minuit.

Romero, C. (2007). « Pour une définition générale de l'intensité dans le langage ». Travaux de linguistique, $\mathrm{n}^{\circ}$ 54, 57-68. DOI : 10.3917/t1.054.0057. URL : https://www.cairn.info/revuetravaux-de-linguistique-2007-1-page-57.htm.

Van De Velde, D. (1995). Le spectre nominal. Dès noms de matières aux noms d'abstractions. Louvian / Paris : Editions Peeters.

Wierzbicka, A. (1971). «Porównanie - gradacja - metafora ». In : Pamiętnik Literacki, 62/4, 127-147. 